\title{
Searching for the role of protein phosphatases in eukaryotic microorganisms
}

A.M. da-Silva ${ }^{1}$, P.D.A. Zapella ${ }^{1}$, L.P.M. Andrioli ${ }^{1}$, R.B. Campanhã ${ }^{1}$, L.C. Fiorini ${ }^{1}$, L.C. Etchebehere ${ }^{1}$,

J.C. da-Costa-Maial† and H.F. Terenzi ${ }^{2}$

\author{
${ }^{1}$ Departamento de Bioquímica, Instituto de Química, \\ Universidade de São Paulo, São Paulo, SP, Brasil \\ ${ }^{2}$ Departamento de Biologia, Faculdade de Filosofia, Ciências e \\ Letras de Ribeirão Preto, Universidade de São Paulo, \\ Ribeirão Preto, SP, Brasil
}

\section{Correspondence \\ A.M. da-Silva \\ Departamento de Bioquímica \\ Instituto de Q uímica, USP \\ Av. Prof. Lineu Prestes, 748 \\ 05509-900 São Paulo, SP \\ Brasil \\ Fax: +55-11-815-5579 \\ E-mail: almsilva@ quim.iq.usp.br \\ tIn memoriam \\ The present address of L.C. Etchebe- here is Núcleo de Pesquisa e Ensino em Bioquímica, Instituto de Ciências Exatas e Biológicas, Universidade Federal de O uro Preto, O uro Preto, MG, Brasil.}

Presented at the I International Symposium on "Signal Transduction and Gene Expression in Cell Proliferation and Differentiation", São Paulo, SP, Brasil,

August 31-September 2, 1998.

Research supported by FAPESP (No. 96/2902-4) and PADCT/CNPq (No. 62.0183/94.2). A.M. da-Silva and H.F. Terenzi were partially supported by CN Pq. P.D.A. Zapella and L.C. Fiorini are graduate fellows supported by FAPESP. L.P.M. Andrioli is a graduate fellow supported by CNPq. R.B. Campanhã was the recipient of a post-doctoral fellowship from FAPESP and L.C. Etchebehere was a research fellow supported by CN Pq.

\begin{abstract}
Preference for specific protein substrates together with differential sensitivity to activators and inhibitors has allowed classification of serine/threonine protein phosphatases (PPs) into four major types designated types 1, 2A, 2B and 2C (PP1, PP2A, PP2B and PP2C, respectively). Comparison of sequences within their catalytic domains has indicated that PP1, PP2A and PP2B are members of the same gene family named PPP. On the other hand, the type $2 \mathrm{C}$ enzyme does not share sequence homology with the PPP members and thus represents another gene family, known as PPM. In this report we briefly summarize some of our studies about the role of serine/threonine phosphatases in growth and differentiation of three different eukaryotic models: Blastocladiella emersonii, Neurospora crassa and Dictyostelium discoideum. Our observations suggest that PP2C is the major phosphatase responsible for dephosphorylation of amidotransferase, an enzyme that controls cell wall synthesis during Blastocladiella emersonii zoospore germination. We also report the existence of a novel acid- and thermo-stable protein purified from Neurospora crassa mycelia, which specifically inhibits the PP1 activity of this fungus and mammals. Finally, we comment on our recent results demonstrating that Dictyostelium discoideum expresses a gene that codes for PP1, although this activity has never been demonstrated biochemically in this organism.
\end{abstract}

Key words

- Protein phosphatases

- Blastocladiella emersonii

\section{Introduction}

In recent years, the participation of protein phosphatases in the dynamic process of protein phosphorylation has gained considerable interest. These enzymes are generally divided into two main groups according to the phosphoamino acid hydrolyzed. Serine/ threonine protein phosphatases (PPs) attack phosphoserine/threonine and tyrosine phosphatases (PTPs) hydrolyze phosphotyrosine.
A subfamily of PTPs, known as dual specificity phosphatases, comprises enzymes that hydrolyze both phosphoserine/threonine and phosphotyrosine. This difference in substrate specificity between PP and PTP groups reflects their tridimensional structures and distinct catalytic mechanisms (1-3).

Specificity towards protein substrates together with differential sensitivity to activators and inhibitors has permitted the classification of PPs into four major types desig- 
nated types 1, 2A, 2B and 2C (PP1, PP2A, PP2B and PP2C, respectively) (4). Amino acid sequence comparisons of their catalytic domains have demonstrated that PP1, PP2A and $\mathrm{PP} 2 \mathrm{~B}$ are members of the same gene family, named PPP. Novel phosphatases, such as PP4, PP5 and PP6, are being identified by molecular cloning, rapidly expanding the PPP family. On the other hand, the type $2 \mathrm{C}$ enzyme shares no sequence homology with the PPP family and represents another gene family, known as PPM $(5,6)$.

Sensitivity to okadaic acid is one of the many biochemical criteria used to identify each type of serine/threonine phosphatase. PP2A, PP4, PP6 and PP5 are inhibited by ten-fold lower concentrations of okadaic acid than those required to inhibit PP1. Even though PP2B, a calcium/calmodulin-activated phosphatase, belongs to the PPP family, it is poorly inhibited by okadaic acid. Enzymes of the PPM family such as PP2C, a magnesium-dependent enzyme, are not affected by okadaic acid $(1,2,4,5)$.

Both PP1 and PP2A exist as multimeric holoenzymes in vivo, where a limited number of catalytic subunits are associated with a variety of regulatory subunits. The latter are believed to modulate catalytic activity and substrate specificity and to act as determinants of subcellular localization of the catalytic subunits $(7,8)$.

The function of PPs in general metabolism, growth and differentiation has been addressed using molecular genetics, principally in yeast and Drosophila. In parallel, the use of some natural toxins, such as okadaic acid, which can act in vivo as potent and specific protein phosphatase inhibitors, has uncovered important physiological roles for these enzymes in different organisms $(1,5,7)$.

In this report we review some of the studies of our laboratory about the participation of serine/threonine phosphatases in growth and differentiation in three different eukaryotic microorganisms: Blastocladiella emersonii, a unicellular aquatic fungus (9),
Neurospora crassa, a filamentous fungus (10), and Dictyostelium discoideum, a microorganism which represents the evolutionary transition between unicellular and multicellular forms of life (11).

\section{PP2C and its role in cell wall biosynthesis during germination in Blastocladiella emersonii}

The initial 20 min of germination by this fungus do not require protein synthesis. Among the many changes leading the zoospore to transform into a round cell, the synthesis of a thin cell wall made of chitin is most significant. In contrast, the conversion of the round cell into a germling does require protein synthesis, which depends on zoospore-stored mRNAs. The germling is able to proliferate and finally differentiate, generating new zoospores. In vitro, potassium, sodium or cyclic AMP triggers germination $(9,12)$.

Some years ago we demonstrated that in the presence of okadaic acid, zoospores form a round cell but cannot produce germlings. Using a chitin-specific fluorescent dye, it was possible to demonstrate that okadaic acid does not inhibit cell wall synthesis (13). Previous work supported the idea that the regulation of cell wall synthesis was mainly at the level of amidotransferase, the first enzyme in the biosynthetic pathway responsible for the synthesis of UDP-N-acetylglucosamine (UDP-GlcNAc), the substrate for chitin synthesis. When this enzyme is phosphorylated by a unknown kinase it is sensitive to inhibition by the final product of the pathway, UDP-GlcNAc, already present in zoospores. Dephosphorylation of amidotransferase allows it to escape from inhibition by UDP-GlcNAc and the pathway can go on to provide enough substrate for the synthesis of chitin $(12,13)$.

Dephosphorylation of amidotransferase can be measured through the loss of UDPGlcNAc inhibition. When zoospore extracts 
containing phosphorylated amidotransferase are preincubated with partially purified $\mathrm{PP} 2 \mathrm{~A}$ and $\mathrm{PP} 2 \mathrm{C}$ in the presence of okadaic acid, EDTA and EGTA almost no dephosphorylation of the enzyme can be observed after up to $60 \mathrm{~min}$ preincubation. However, if okadaic acid is not included in the preincubation period some dephosphorylation occurs. Maximum dephosphorylation is observed when the extracts are incubated in the presence of magnesium, which activates PP2C (13). In addition, we have observed that partially purified amidotransferase can be dephosphorylated when preincubated with PP2C purified by us from Blastocladiella zoospores and the dephosphorylation, as expected, is not affected by okadaic acid and is dependent on magnesium (Campanhã RB, unpublished results).

These results lead us to propose that PP2C is the major phosphatase responsible for amidotransferase dephosphorylation during zoospore germination (Figure 1). Dephosphorylation of amidotransferase releases it from the inhibition by UDP-GlcNAc, allowing the enzyme to catalyze the first reaction in the pathway for UDP-GlcNAc synthesis. Recently, we demonstrated a direct in vitro inhibition of PP2C by UDP-GlcNAc (Campanhã RB, unpublished results). Thus, UDPGlcNAc plays a dual role in hexosamine biosynthesis and chitin synthesis in Blastocladiella zoospores. It not only inhibits the phosphorylated form of the enzyme $(12,13)$ but it also prevents its dephosphorylation by direct inhibition of PP2C.

\section{A novel PP1 inhibitor from Neurospora crassa}

The major active serine/threonine phosphatase in Neurospora crassa is PP1, which accounts for about $60 \%$ of the total activity. PP2A, PP2B and PP2C activities are also demonstrable in crude extracts (14-16). During our attempts to characterize these activities in Neurospora, we observed that a short period of incubation of crude extracts with trypsin resulted in a slight increase in PP1 activity, which suggested that PP1 catalytic subunits were complexed with inhibitory proteins, as observed for mammalian PP1 $(4,17)$. These inhibitory proteins have been described for mammal and invertebrate cells, and appear to modulate PP1 activity as well as its subcellular localization (8).

These observations led us to search for PP1 inhibitory proteins in crude Neurospora mycelial extracts. As reported elsewhere (14), we partially purified a protein fraction that was highly effective in inhibiting Neurospora PP1 activity. This acid- and thermostable inhibitory protein was further purified and its inhibitory properties were confirmed using partially purified Neurospora crassa PP1 and commercial rabbit recombinant PP1. The Neurospora crassa purified PP1 inhibitor $(\mathrm{INc})$ is specific for the PP1 catalytic subunit, since it does not inhibit the PP2A catalytic subunit purified from Dictyostelium discoideum, or partially purified PP2A from Neurospora crassa or rat liver (14; Figure 2).

Recently we succeeded in demonstrating the inhibitory activity of $I N c$ against $N$. crassa recombinant PP1 expressed in E. coli (Zapella

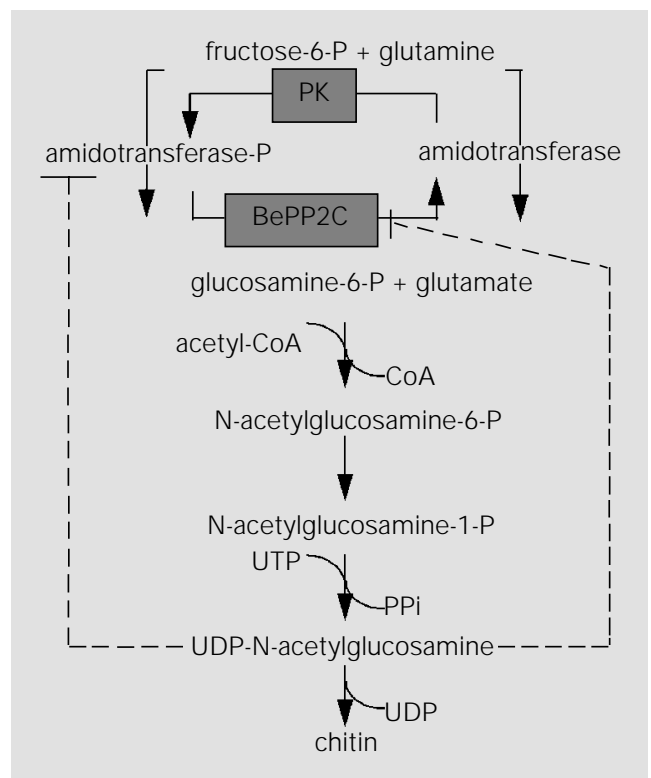

Figure 1 - Control of chitin synthesis in Blastocladiella emersonii. Both forms of amidotransferase catalyze the synthesis of glucosamine-6-phosphate. However, only the phosphorylated form (amidotransferase- $P$ ) is inhibited by UDP-GlcNAc (dashed line on the left side). UDPGlcNAc also prevents amidotransferase dephosphorylation by direct inhibition of PP2C (dashed line on the right side). PK, Protein kinase; BePP2C PP2C purified from Blastocladiella. This scheme was modified from Ref. 12 to include PP2C as the major enzyme responsible for amidotransferase dephosphorylation. 
Figure 2 - Neurospora crassa PP1 inhibitor (INc) is specific against PP1 activity when phosphorylase phosphatase is used as substrate to assay this enzyme. Serial dilutions of purified INc were assayed as described (14) against Neurospora partially purified PP1 (black triangle), rabbit recombinant PP1 (open triangle) and PP2A catalytic subunit purified from Dictyostelium discoideum (black circle).

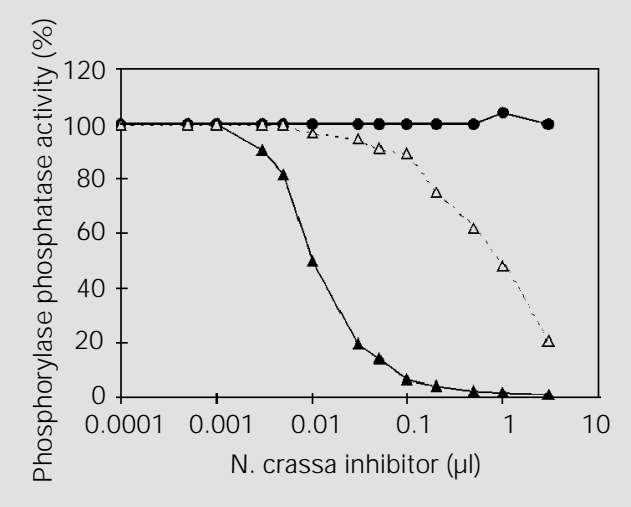

PDA, unpublished results). In order to perform this kind of experiment we isolated a 2333-base pair cDNA corresponding to $\mathrm{Neu}$ rospora PP1 (GenBank accession number AF049853), which probably is related to a PP1 catalytic subunit that was recently purified from this fungus (16). The $N$. crassa recombinant PP1 has been an important tool for in vitro binding assays to INc. In addition PP 1 cDNA is also being used as bait in yeast two hybrid assays to search for $N$. crassa PP1 modulatory subunits.

To our knowledge, no other fungal PP1 inhibitor has been identified by biochemical approaches thus far. The biochemical characteristics of INC are currently being investigated in depth and preliminary data on its amino acid sequence suggests that $\mathrm{I} N c$ is a novel protein.

\section{D ictyostelium cells express a PP1-like gene}

Many of the major groups of known protein kinases have been identified in Dictyostelium and their roles in controlling growth and development are under investigation (1820). In contrast, almost nothing is known about the protein phosphatases that participate in these processes. Previous work demonstrated that Dictyostelium extracts contain PP2A, PP2B and PP2C catalytic activities with biochemical properties similar to those of their mammalian and yeast counterparts
(21). In contrast, no type 1 protein phosphatase (PP1) was detected during Dictyostelium life cycle (21) even though PP1 has been shown to be present in all other organisms analyzed.

Increasing efforts are been devoted to the identification of Dictyostelium genes that code for protein phosphatases. Such genes should be important tools to better understand the role of these enzymes in Dictyostelium growth and development. A complete cDNA clone for PP2B was isolated but the role played by this enzyme in Dictyostelium is still unclear $(22,23)$. More recently a novel PP2C gene found in Dictyostelium was described as essential for its cell type differentiation (24).

Through the use of PCR with a pair of degenerated oligonucleotide primers derived from conserved residues in the catalytic core of the PPP family, it has been possible to identify different members of this gene family in many organisms $(5,25)$ including Dictyostelium discoideum (26). Using this approach we have isolated genomic DNA sequences encoding putative $\mathrm{PP}$ catalytic subunits. Amplified DNA fragments of the expected size were cloned and sequenced. Two of the selected clones were chosen for further analysis. One with high similarity to PP1 and the other with high similarity to PP4. These clones were used as probes to screen a cDNA library derived from Dictyostelium vegetative cells. Full length cDNAs for both catalytic subunits were isolated (Andrioli LPM and Fiorini LC, unpublished results), revealing that Dictyostelium cells express genes that code for PP1 and for PP4 catalytic subunits. The sequence of the cDNA that encodes PP1 (GenBank accession number AF020537) showed that Dictyostelium enzyme is similar to PP1 from other organisms. Moreover Dictyostelium recombinant PP1 expressed in E. coli is active and shows biochemical properties similar to those of a typical PP1 (Andrioli LPM, unpublished results). PP1 activity seems to be essential for 
growth of Dictyostelium cells since we have failed after many attempts to knock out the only PP1 gene that exists in the Dictyostelium genome (Andrioli LPM, unpublished results). Taken together, these observations contrast with the failure in detecting biochemically PP1 activity in Dictyostelium cell extracts. Hence the question is why is this enzyme activity not demonstrable in Dicty- ostelium cell extracts? One possibility is the existence of an inhibitory subunit tightly associated with the PP1 catalytic subunits in the cell extracts. The availability of cDNA clones coding for protein phosphatases paves the way for a molecular genetic analysis of the role of this class of enzymes in Dictyostelium growth and development.

\section{References}

1. Wera S \& Hemmings BA (1995). Serine/ threonine protein phosphatases. Biochemical J ournal, 311: 17-29.

2. Hunter T (1995). Protein kinases and phosphatases: the yin and yang of protein phosphorylation and signaling. Cell, 80: 225-236.

3. Denu J M, Stuckey J A, Saper MA \& Dixon JE (1996). Form and function in protein dephosphorylation. Cell, 87: 361-364.

4. Cohen $P$ (1989). The structure and regulation of protein phosphatases. Annual Review of Biochemistry, 58: 453-508.

5. Cohen PT (1997). Novel protein serine/ threonine phosphatases: variety is the spice of life. Trends in Biochemical Sciences, 22: 245-251.

6. Villafranca J E, Kissinger CR \& Parge HE (1996). Protein serine/threonine phosphatases. Current Opinion in Biotechnology, 7: 397-402.

7. Depaoli-Roach AA, Park IK, Cerovsky V, Csortos C, Durbin SD, Kuntz MJ, Sitikov A, Tang PM, Verin A \& Zolnierowicz $S$ (1994). Serine/threonine protein phosphatases in the control of cell function. Advances in Enzyme Regulation, 34: 199224.

8. Hubbard MJ \& Cohen P (1993). On target with a new mechanism for the regulation of protein phosphorylation. Trends in Biochemical Sciences, 18: 172-177.

9. Barstow WE \& Freshour GD (1987). Blastocladiella emersonii. In: Fuller MS \& J aworski A (Editors), Zoosporic Fungi in Teaching and Research. Southeastern Publishing Corporation, Athens.

10. Metzenberg RL \& Glass NL (1990). Mating type and mating strategies in Neurospora. BioEssays, 12: 53-59.

11. Baldauf SL \& Doolittle WF (1997). Origin and evolution of the slime molds. Proceedings of the National Academy of Sci- ences, USA, 94: 12007-12012.

12. da Costa Maia J C (1994). Hexosamine and cell wall biogenesis in the aquatic fungus Blastocladiella emersonii. FASEB J ournal, 8: 848-853.

13. Etchebehere LC, Simon MN, Campanhã RB, Zapella PDA, Veron $M \&$ da Costa Maia JC (1993). Developmental regulation of hexosamine biosynthesis by protein phosphatases $2 \mathrm{~A}$ and $2 \mathrm{C}$ in Blastocladiella emersonii. J ournal of Bacteriology, 175: 5022-5027.

14. Zapella PDA, da Silva AM, da Costa Maia J C \& Terenzi HF (1996). Serine/threonine protein phosphatase 1 inhibitor from Neurospora crassa. Brazilian J ournal of Medical and Biological Research, 29: 599-604.

15. Prokisch $H$, Yarden $O$, Dieminger $M$, Tropschug M \& Barthelmess IB (1997). Impairment of calcineurin function in Neurospora crassa reveals its essential role in hyphal growth, morphology and maintenance of the apical $\mathrm{Ca}^{2+}$ gradient. Molecular and General Genetics, 256: 104-114.

16. Szoor B, Dombradi V, Gergely P \& Feher Z (1997). Purification and characterization of the catalytic subunit of protein phosphatase 1 from Neurospora crassa. Acta Biologica Hungarica, 48: 289-302.

17. Shenolikar S \& Nairn AC (1991). Protein phosphatases: recent progress. Advances in Second Messenger and Phosphoprotein Research, 23: 1-121.

18. Maeda M (1997). Aggregation-related protein kinases in Dictyostelium, MAP-kinase ERK2 and CAMP-dependent protein kinase PKA. In: Maeda $Y$, Inouye $K$ \& Takeuchi I (Editors), Dictyostelium. A Model System for Cell and Developmental Biology. Universal Academy Press, Tokyo.

19. Loomis WF (1998). Role of PKA in the timing of developmental events in Dictyostelium cells. Microbiology and Molecu- lar Biology Reviews, 62: 684-694.

20. Souza GM, Lu S \& Kuspa A (1998). YakA, a protein kinase required for the transition from growth to development in Dictyostelium. Development, 125: 2291-2302.

21. Simon MN, Winckler T, Mutzel R, Véron M \& da Costa Maia J C (1992). Serine/ threonine protein phosphatases in Dictyostelium discoideum: No evidence for type I activity. Biochemical and Biophysical Research Communications, 184: 1142-1151.

22. Dammann $H$, Hellstern $S$, Husain $Q \&$ Mutzel R (1996). Primary structure, expression and developmental regulation of a Dictyostelium calcineurin A homologue. European J ournal of Biochemistry, 238: 391-399.

23. Hellstem S, Dammann $\mathrm{H}$, Husain $\mathrm{Q} \&$ Mutzel R (1997). Overexpression, purification and characterization of Dictyostelium calcineurin A. Research in Microbiology, 148: 335-343.

24. Aubry L \& Firtel RA (1998). Spalten, a protein containing Galpha-protein-like and PP2C domains, is essential for cell-type differentiation in Dictyostelium. Genes and Development, 12: 1525-1538.

25. Barton GJ , Cohen PT \& Barford D (1994). Conservation analysis and structure prediction of the protein serine/threonine phosphatases. Sequence similarity with diadenosine tetraphosphatase from Escherichia coli suggests homology to the protein phosphatases. European J ournal of Biochemistry, 220: 225-237.

26. Haribabu B \& Dottin RP (1991). Homology cloning of protein kinase and phosphoprotein phosphatase sequences of Dictyostelium discoideum. Developmental Genetics, 12: 45-49. 